\title{
RNAi-mediated downregulation of radiation-induced MMP-9 leads to apoptosis via activation of ERK and Akt in IOMM-Lee cells
}

\author{
VENKATESWARA RAO GOGINENI ${ }^{1}$, ODYSSEAS KARGIOTIS ${ }^{4}$, JEFFREY D. KLOPFENSTEIN ${ }^{2}$, \\ MEENA GUJRATI ${ }^{3}$, DZUNG H. DINH ${ }^{2}$ and JASTI S. RAO ${ }^{1,2}$ \\ Departments of ${ }^{1}$ Cancer Biology and Pharmacology, ${ }^{2}$ Neurosurgery and ${ }^{3}$ Pathology, \\ University of Illinois College of Medicine at Peoria, One Illinis Drive, Peoria, IL 61605, USA; \\ ${ }^{4}$ Neurosurgical Institute, University of Ioannina Medical School, Ioannina, Greece
}

Received June 25, 2008; Accepted September 15, 2008

DOI: 10.3892/ijo_00000143

\begin{abstract}
Patients afflicted with meningiomas are most often treated with radiation therapy followed by surgical resection. However, resistance to radiation treatment has been well documented among different cancers of the brain. In this study, we demonstrate that the malignant meningioma cells (IOMM-Lee cells) overexpress MMP-9 at both the mRNA and protein levels after radiation treatment. We confirmed an increase in the invasive potential of irradiated cells through spheroid migration and Matrigel invasion assays. Knockdown of MMP-9 using an adenoviral siRNA construct blocked MMP-9 expression, reduced the invasive nature of cells, and subsequently led to apoptosis. Western blot analysis revealed the activation of ERK, Akt and Fas as well as a decrease in c-JUN levels. Cleavage of PARP and TUNEL-positive characteristics confirmed apoptotic cell death in Ad-MMP-9 infected cells. Treatment with U0126 and transfection with dominant negative ERK plasmid resulted in the decreased phosphorylation of ERK and Akt. Ectopic expression of HA myr-Akt was found to be associated with an increase in pERK, and treatment with LY294002 was shown to block the phosphorylation of Akt and ERK with the restoration of c-JUN. In conclusion, our data suggest that radiation increases MMP-9 expression and the invasive nature of IOMM-Lee cells, both of which can be reversed with siRNA-mediated downregulation of MMP-9, which leads to ERK and Akt-mediated apoptosis.
\end{abstract}

Correspondence to: Dr Jasti S. Rao, Department of Cancer Biology and Pharmacology, University of Illinois College of Medicine, One Illini Drive, Peoria, IL 61605, USA

E-mail: jsrao@uic.edu

Key words: meningioma, IOMM-Lee, MMP-9, siRNA, radiation, apoptosis, extracellular signal regulated kinase, protein kinase B (Akt)

\section{Introduction}

Meningiomas are the most common benign tumors of the brain. They originate from the arachnoidal cells of the meninges and constitute approximately $20 \%$ of all primary intracranial tumors, with an approximate annual incidence of 6 per 100,000 (1). The majority of patients diagnosed with a meningioma undergo a surgical resection to reduce neurological symptoms. Complete surgical resection is often curative, but for patients with tumors in surgically inaccessible locations or patients of advanced age, radiotherapy is the treatment modality of choice. Besides the fact that meningiomas grow slowly and have a low mitotic potential, radiation therapy has been reported to be promising in regressing tumor growth after incomplete resection $(2,3)$ after recurrence (4); and when tumor histology reveals atypia or anaplasia (5-7). However, the development of aggressive characteristics in different tumors after radiotherapy has been widely reported. Reports of radioresistance and radiation-induced invasive and angiogenic characteristics in gliomas and other brain cancers have been gaining significance $(8,9)$. In addition, radiotherapy of other types of brain tumors has been shown to induce the spontaneous development of atypical and anaplastic meningiomas $(10,11)$.

Invasive and metastatic mechanisms are complex and recruit numerous classes of proteins including cadherins, integrins, extracellular proteases and angiogenic factors. Among them, matrix metalloproteinases (MMPs) play a key role in the degradation of extracellular matrix (ECM) proteins. In almost all types of human cancer, augmented MMP expression and activity are associated with advanced tumor stage and poor survival. Elevated plasma levels of MMP-9 have been found in lung cancer, breast cancer and liver cancer during radiotherapy $(12,13)$. Moreover, MMP inhibitors have been shown to block radiation-induced invasiveness of human pancreatic cancer cells (14).

RNAi-mediated knockdown of components that are essential for cancer cell survival, metastasis, angiogenesis and resistance to crisis have been widely studied. Recent studies have demonstrated the effectiveness of an adenoviral construct 
with MMP-9 siRNA to knockdown MMP-9 expression and induce apoptosis in medulloblastoma cell lines $(15,16)$. Molecular analysis of the signaling cascades that lead to apoptosis is essential for delineating cell death events and to develop novel cancer therapeutics. The involvement of extracellular signal-regulated protein kinases 1 and 2 (ERK1/2) and protein kinase $\mathrm{B}$ (PKB or Akt) in cell survival pathways has been well characterized. However, recent evidence suggests the pro-apoptotic role of ERK1/2 in neuronal and renal epithelial cells upon exposure to oxidative stress, toxics and upon deprivation of growth factors $(17,18)$. Akt also plays an apoptotic role in Fas-mediated cell death in epidermal and melanoma cell lines through a mechanism that involves transcriptional activation of the Fas receptor (19-21).

In this study, we are interested in understanding the effect of radiation on MMP-9 in a meningioma cell line. Therefore, we evaluated the effect of MMP-9 siRNA on irradiated IOMM-Lee cells. Our results show that Ad-MMP-9 induced apoptosis via phosphorylation of ERK and Akt through a Fas-mediated mechanism.

\section{Materials and methods}

Cell culture. We used the human meningioma cell line IOMM-Lee for the present study (kindly provided by Dr Ian E. McCutcheon, University of Texas M.D. Anderson Cancer Center, Houston, TX). The cells were maintained in Dulbecco's modified Eagle's medium (Mediatech, Herndon, VA) supplemented with $10 \%$ fetal bovine serum, $100 \mu \mathrm{g} / \mathrm{ml}$ streptomycin and $100 \mathrm{U} / \mathrm{ml}$ penicillin (Invitrogen, Carlsbad, $\mathrm{CA}$ ) in a humidified atmosphere containing $5 \% \mathrm{CO}_{2}$ at $37^{\circ} \mathrm{C}$. The primary antibodies used for this study were anti-ERK, anti-pERK, anti-c-JUN, anti-Fas (Santa Cruz Biotechnologies, Santa Cruz, CA), anti-Akt, anti-pAkt, anti-pc-JUN (ser243), anti-pc-JUN(73) and anti-GAPDH (Cell Signaling, Boston, MA) and HRP-conjugated secondary antibodies from Biomeda (Foster City, CA). Specific inhibitors of MEK1/2 (U0126) and phosphatidylinositol 3-kinase (PI3K) (LY294002) were purchased from Calbiochem (San Diego, CA). The expression vector of constitutively active Akt (HA-Akt-myr) was purchased from Addgene, MA (Addgene, Plasmid 10841).

Adenoviral siRNA constructs and infection. The adenoviral siRNA constructs for MMP-9 (Ad-MMP-9) and scrambled vector (Ad-SV) were constructed and amplified as described previously (16). Viral titers were quantified as plaque-forming units/ml following infection of 293 cells. We obtained the following titers for the viruses: Ad-SV (7.6x $10^{11}$ plaqueforming units/ml) and Ad-MMP-9 (5.0x10 11 plaque-forming units $/ \mathrm{ml})$. The amount of infective adenoviral vector per cell (plaque-forming units/cell) in culture media was expressed as multiplicity of infection (MOI). Virus constructs were diluted in serum-free culture media to $50 \mathrm{MOI}$, added to cells, and incubated at $37^{\circ} \mathrm{C}$ for $1 \mathrm{~h}$. The necessary amount of complete medium was then added, and cells were incubated for the desired time periods.

Transfection with plasmids. All transfection experiments were performed with FuGENE $H D$ transfection reagent as per the manufacturer's protocol (Roche Applied Science,
Indianapolis, IN). IOMM-Lee cells were transfected with plasmid constructs containing ERK dominant negative mutant (Dn-ERK) (22) and HA myr Akt. Briefly, plasmid containing either Dn-ERK or HA myr-Akt was mixed with FuGENE $H D$ reagent (1:3 ratio) in $500 \mu 1$ of serum-free medium and left for $30 \mathrm{~min}$ for complex formation. The complex was then added to the plate, which had $2.5 \mathrm{ml}$ of serum-free medium ( $2 \mu \mathrm{g}$ of plasmid/ml of medium). After $6 \mathrm{~h}$ of transfection, complete medium was added and kept for $24 \mathrm{~h}$ and used for further experiments.

Radiation treatment. The RS 2000 Biological Irradiator (Rad Source Technologies, Inc., Boca Raton, FL) X-ray unit, which was operated at $150 \mathrm{kV} / 50 \mathrm{~mA}$, was used for radiation treatments. Cells were infected with Ad-SV or Ad-MMP-9 or transfected with plasmids; a single dose of radiation $(2.5,5$ or $7.5 \mathrm{~Gy}$ ) was given to infected or control IOMM-Lee cells and tumor spheroids (in 96-well plates).

Gelatin zymography. MMP-9 expression levels after AdMMP-9 infection and radiation treatment were analyzed using gelatin zymography. IOMM-Lee cells were infected with either Ad-MMP-9 or Ad-SV; untreated cells were also cultured to serve as the control. After a 24-h incubation period, one set each of infected and uninfected plates were irradiated with $5 \mathrm{~Gy}$ and the serum-containing media from all the plates was replaced with serum-free media. After further incubation for $16 \mathrm{~h}$, conditioned media were collected from the cells and centrifuged to remove cellular debris. Equal amounts of protein were subjected to electrophoresis on $10 \%$ acrylamide gels containing gelatin $(0.5 \mathrm{mg} / \mathrm{ml})$. Gels were stained with amido black (Sigma Aldrich, St. Louis, MO) and gelatinase activity of MMP-9 was visualized as clear bands on a dark blue background at areas corresponding to the molecular weight of the protein.

Reverse transcription PCR. IOMM-Lee cells were infected and irradiated as described above, and total RNA was extracted as described by Chomczynski and Sacchi (23). PCR was performed using a reverse transcription-PCR (RT-PCR) kit (Invitrogen): 35 cycles of denaturation at $94^{\circ} \mathrm{C}$ for $1 \mathrm{~min}$, annealing at $67^{\circ} \mathrm{C}$ for $30 \mathrm{sec}$, and extension at $72^{\circ} \mathrm{C}$ for $90 \mathrm{sec}$. The expected PCR products were visualized using ethidium bromide after resolving on $2 \%$ agarose gels. RT-PCR for glyceraldehyde-3-phosphate dehydrogenase (GAPDH) was performed to normalize input RNA. We used the following primers: sense, 5'-TGGACGATGCCTGCAACGTG-3' and antisense, 5'-GTCGTGCGTGTCCAAAGGCA-3' (MMP-9); sense, 5'-TGAAGGTCGGAGTCAACGGATTTGGT-3' and antisense, 5'-CATGTGGGCCATGAGGTCCACCAC-3' (GAPDH).

Matrigel invasion assay. IOMM-Lee cells were infected with Ad-MMP-9 or Ad-SV and irradiated as described above. After irradiation, cells were trypsinized and $1 \times 10^{5}$ cells were placed into Matrigel-coated transwell inserts with $8-\mu \mathrm{m}$ pore size. Cells were allowed to migrate through the Matrigel for $24 \mathrm{~h}$. Then, cells in the upper chamber were removed by cotton swab. Cells adhered on the outer surface of the transwell which had invaded through the Matrigel were fixed, 

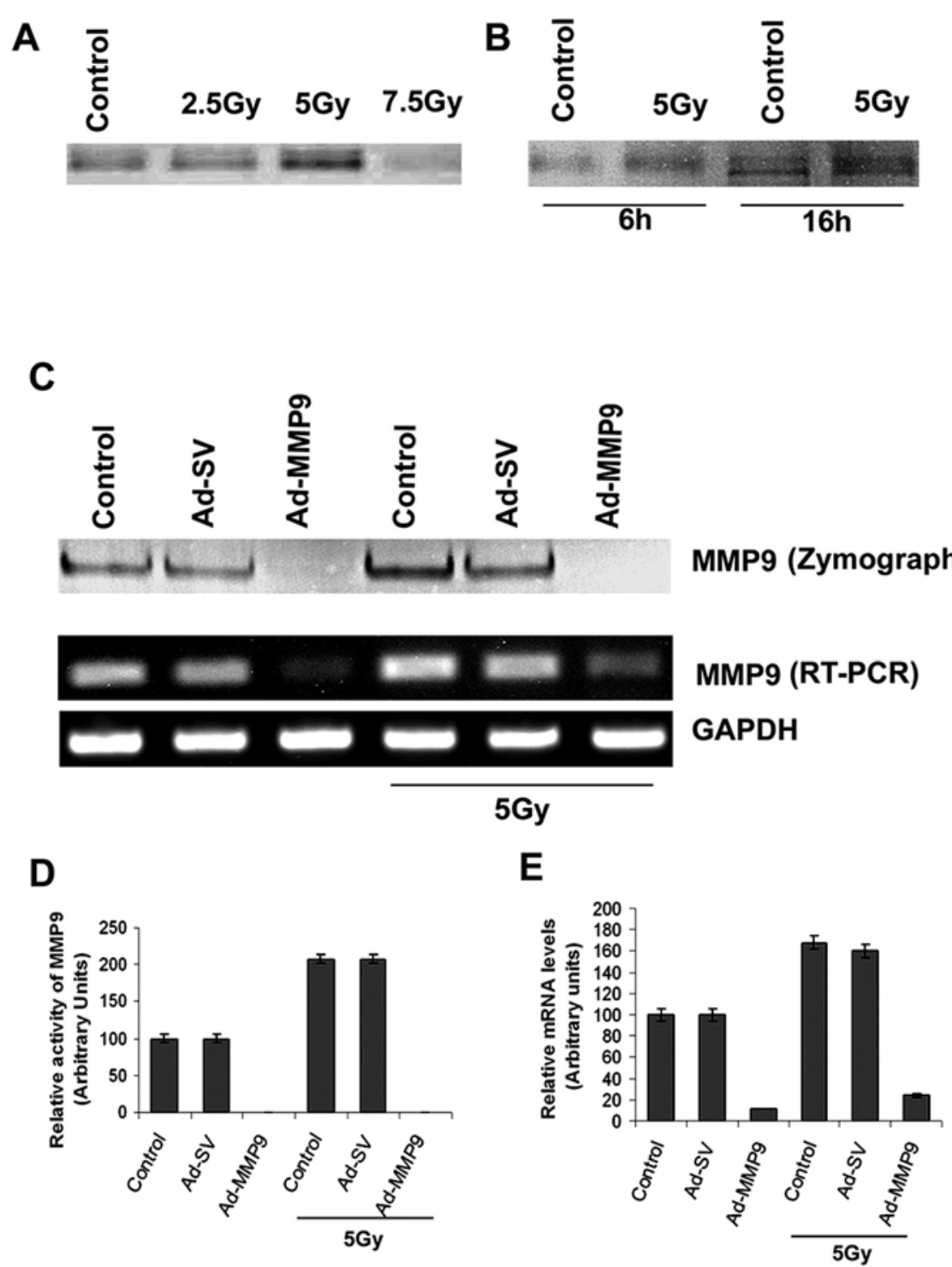

Figure 1. Radiation increases MMP-9 expression in IOMM-Lee cells. (A) Gelatin zymography using the conditioned media from IOMM-Lee cell cultures radiated with 2.5, 5 and $7.5 \mathrm{~Gy}$. (B) Gelatin zymography of the conditioned media from IOMM-Lee cells irradiated with 5 Gy dose for 6- and 16-h time-points against their respective controls. (C) IOMM-Lee cells were infected with 50 MOI of Ad-SV or Ad-MMP-9; 24 h later, cells were irradiated with 5 Gy and incubated in serum-free medium for $16 \mathrm{~h}$. Conditioned medium $(20 \mu \mathrm{g})$ was used for gelatin zymography. Total RNA was extracted as per standard protocols from the cells. Reverse transcription PCR analysis was performed using primers specific for MMP-9 and GAPDH. (D and E) Results from three independent experiments are shown as means \pm SD $(\mathrm{p}<0.05)$. The changes in MMP-9 activity (D) and expression (E) were quantitated and compared with the control values.

stained using the Hema-3 staining kit, and counted under a light microscope as described previously (24).

Western blot analysis. Protein extracts were obtained from the IOMM-Lee cells using Tris-buffered lysis (Tris-buffered saline, $20 \mathrm{mM}$ EDTA, $0.1 \%$ Triton X-100). Cell lysates were also collected from untreated cells that were cultured and maintained under similar conditions (mock). Protein concentration was determined using a bicinchoninic acid procedure (Pierce, Rockford, IL). Equal amounts of protein were then subjected to SDS-PAGE using gels with appropriate percentage of acrylamide followed by transfer of protein to polyvinylidene difluoride membranes (Bio-Rad, Hercules, CA). Membranes were then blocked in 5\% non-fat dry milk in phosphate-buffered saline (PBS) and incubated overnight at $4^{\circ} \mathrm{C}$ with primary antibodies in blocking solution $(1: 1000$ dilution). Membranes were then washed twice (15 min per wash) with T-PBS [Tween-20 (0.1\%), phosphate-buffered solution]. Horseradish peroxidase (HRP)-conjugated secondary antibodies (Biomeda, Burlingame, CA) were used at a 1:2000 concentration. The membranes were developed following an enhanced chemiluminescence protocol (Amersham Biosciences, Piscataway, NJ). The membranes were further probed for GAPDH, which was used as a loading control.

Spheroid migration assay. IOMM-Lee cells labeled with GFP (green fluorescence protein) were cultured at a concentration of $7 \times 10^{4}$ cells per well in $0.5 \%$ agarose-coated 96 -well plates and grown for 2 days at $37^{\circ} \mathrm{C}$ with shaking at 40-60 rpm. The spheroids were infected with Ad-MMP-9 or Ad-SV. 
Untreated spheroids were also maintained under similar conditions for $24 \mathrm{~h}$ to serve as the control. Later, the spheroids were transferred to 8 -well chamber slides and grown in serum-free media for a further $48 \mathrm{~h}$. Migration of the cells from spheroids was recorded with a fluorescence microscope. Spheroid migration was quantified as the relative migration by the tumor cells from the spheroids against the control and scrambled vector-infected cells.

TUNEL assay. To evaluate the apoptotic response of AdMMP-9, we performed the terminal deoxynucleotide transferase (TdT)-mediated biotin-dUTP nick end-labeling technique using the commercially available In Situ Cell Death Detection Kit (Roche Applied Science). Briefly, 5,000 cells were seeded onto the 8 -well chamber slides and infected with 50 MOI of Ad-SV or Ad-MMP-9. Twenty-four hours after infection, the cells were irradiated and incubated for another $16 \mathrm{~h}$. The cells were washed, fixed with $4 \%$ buffered paraformaldehyde, and permeabilized with freshly prepared $0.1 \%$ Triton X-100 containing $0.1 \%$ sodium citrate. The cells were then incubated with TUNEL reaction mixture for $1 \mathrm{~h}$ at $37^{\circ} \mathrm{C}$ in a humidified chamber. The slides were washed three times with PBS, and the incorporated biotin-dUTP was detected under a fluorescent microscope. Cell death was quantified as the relative percent of apoptosis compared to the controls.

\section{Results}

Radiation induces MMP-9 activity and expression. The dose-dependent studies with radiation ranging from 2.5 to 7.5 Gy on IOMM-Lee cells showed an increase in MMP-9 activity at 5 Gy but a further increase in the dose led to the loss of activity (Fig. 1A). This effect could be due to protein degradation at higher doses of radiation. Although a dose of 5 Gy induced MMP-9 activity $6 \mathrm{~h}$ after treatment, the activity was more prominent after $16 \mathrm{~h}$ (Fig. 1B). Gelatin zymography and reverse transcription PCR analysis revealed that significant increase in MMP-9 with irradiated cells compared to the non-irradiated cells (Fig. 1C). Densitometric analysis revealed a significant increase in MMP-9 activity and RNA levels in the irradiated cells (Fig. 1D and E).

Ad-MMP-9 reduces radiation-induced expression of MMP-9. To evaluate the efficiency of Ad-MMP-9 in the knockdown of MMP-9, the cells were infected with 50 MOI of AdMMP-9 or Ad-SV with and without irradiation. As shown in the zymography and reverse transcription $\mathrm{PCR}$ results (Fig. 1C), Ad-MMP-9 treatment at 50 MOI significantly reduced the activity of MMP-9 and mRNA levels in both non-irradiated and irradiated cells, thereby showing the efficacy of RNAi-mediated knockdown of MMP-9 (Fig. 1D and E). Activity and mRNA levels in the cells infected with Ad-SV remained similar to their respective controls.

Ad-MMP-9 reduces radiation-induced migration and invasion. Noting that irradiation induced MMP-9 expression but infection with Ad-MMP-9 reduced MMP-9 activity, we next analyzed the invasive and migration abilities of the IOMM-Lee cells. Spheroid migration assay with IOMM-Lee-
GFP cells showed an increase in the migration properties of the cells in the irradiated treatment groups as compared to the controls. However, migration of the cells from Ad-MMP-9infected spheroids was markedly diminished both in the irradiated and non-irradiated cells (Fig. 2A). The increase in migration was quantified as the relative migration of the treated cells against the controls (Fig. 2B). Radiation treatment was shown to induce $>50 \%$ increase in the migration of IOMM-Lee cells and decreased almost $>98 \%$ of the cells infected with Ad-MMP-9. Matrigel invasion assay revealed a similar trend with irradiated cells showing more invasive potential compared to the non-irradiated cells. Ad-MMP-9infected cells in both irradiated and non-irradiated groups showed very poor invasion (Fig. 2C). Quantitative determination of invasion confirmed that IOMM-Lee cells infected with Ad-MMP-9 invaded only $<10 \%$ with and without radiation (Fig. 2D) compared to controls and Ad-SV treated cells.

Ad-MMP-9 infection induces apoptosis. Infection of IOMMLee cells with Ad-MMP-9 induced a marked change in the morphological characteristics of the cells within the experimental time period (data not shown). Based on these observations, we performed Western blot analysis for the cleavage of PARP and TUNEL assay for in situ cell death. TUNEL assay revealed that the majority of the Ad-MMP-9infected cells were fluorescent in both irradiated and nonirradiated groups (Fig. 3A). Cell death was quantified as percent of apoptotic cells against the controls. Fig. 3B shows that $\sim 80-90 \%$ of apoptotic cells in Ad-MMP-9 infected cells compared to controls and Ad-SV infected cells. We found that Ad-MMP-9 infection caused cleavage of PARP, which is a downstream effector of caspases. As shown in Fig. 3C, the $85-\mathrm{kDa}$ cleaved fragment of PARP-1 was detected in AdMMP-9-treated cells, but not in control and Ad-SV-infected cells.

Ad-MMP-9 infection activates ERK and Akt. We next investigated the signal transduction mechanism of apoptosis in Ad-MMP-9-infected cells. Cell lysates were subjected to Western blot analysis and probed with antibodies against phospho-ERK, phospho-Akt, and their respective total forms. Ad-MMP-9 infection increased phospho-ERK and phosphoAkt as compared to the control and Ad-SV-infected cells with and without radiation. But there was no change in total ERK and Akt levels in treated and non-treated cells with and without radiation (Fig. 4A). Densitometric analysis indicated $>9$-fold increase in pERK and $>15$-fold increase in pAkt in the Ad-MMP-9-infected cells (Fig. 4B and C) compared to control and Ad-SV infected cells. Similar trend was observed in Ad-MMP-9 infected cells with and without irradiation in relation to these molecules.

MEK1/2 inhibitor and Dn-ERK plasmid reduce the activity of $E R K$ and Akt. To investigate the interactions between Akt and ERK, we treated the Ad-MMP-9 infected cells with U0126; the cell lysates were subjected to Western blot analysis. In addition, we transiently transfected IOMM-Lee cells with a dominant negative mutant of ERK (Dn-ERK; kinase-dead mutants of ERK-1) prior to Ad-MMP-9 infection and analyzed 
A
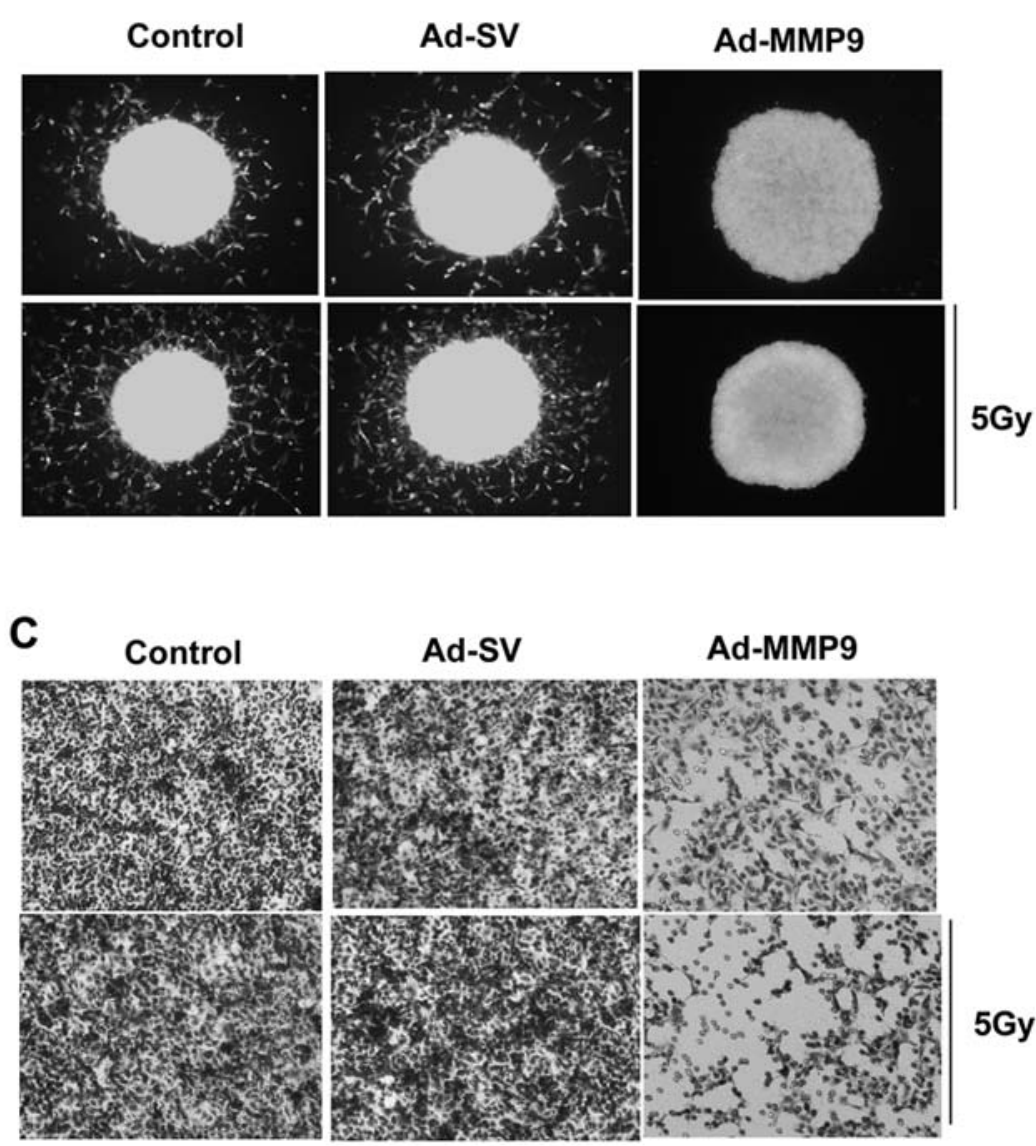

B

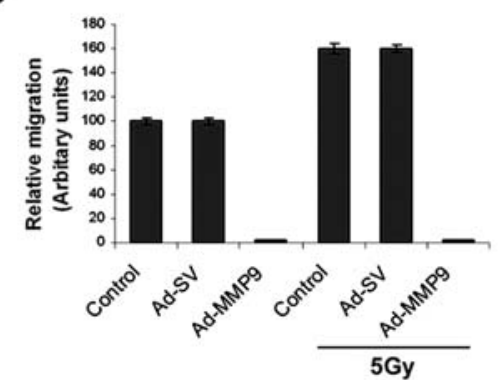

D

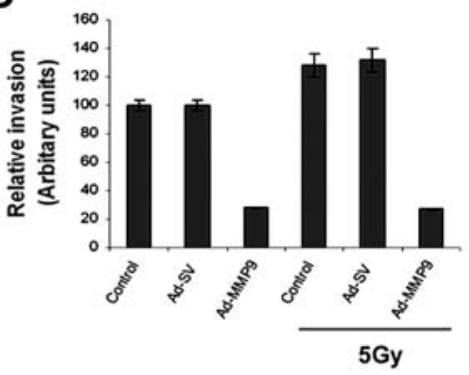

Figure 2. Knockdown of MMP-9 with Ad-MMP-9 reduces spheroid migration and invasion. (A) Fluorescent-labeled IOMM-Lee cells were cultured in 96-well low attachment plates at a concentration of $7 \times 10^{4}$ and spheroids were allowed to grow for $24 \mathrm{~h}$ at $37^{\circ} \mathrm{C}$ with shaking at $40-60 \mathrm{rpm}$. The spheroids were then infected with Ad-SV or Ad-MMP-9 and irradiated after $24 \mathrm{~h}$. Untreated spheroids were also maintained to serve as the control (mock). Immediately after radiation treatment, the spheroids were transferred to 8-well chamber slides and maintained for another $24 \mathrm{~h}$ in serum-free media. Spheroid migration was analyzed by taking pictures using a fluorescent microscope. (B) Cell migration from the spheroids was quantified as the distance cells migrated from the spheroids. Values are mean \pm SD from three different experiments ( $<<0.05)$. (C) IOMM-Lee cells were infected with Ad-SV or Ad-MMP-9 and irradiated at 5 Gy after $24 \mathrm{~h}$ of infection. Untreated (mock) were also maintained to serve as the control. After irradiation, the cells were trypsinized and $1 \times 10^{5}$ cells from each treatment group and controls were cultured in the upper chamber of a transwell insert coated with Matrigel (1 mg/ml). (D) We counted the number of cells in three different fields for each sample. The percentage invasion of cells treated with Ad-MMP-9 was analyzed and compared with the untreated (mock) cells. The graph represents the percentage invasion shown by cells infected with Ad-MMP-9 in comparison with untreated cells (mock). Values are mean \pm SD from three different experiments $(\mathrm{p}<0.05)$.

its effect on the phosphorylation of Akt. U0126 completely inhibited the phosphorylation of ERK and Akt in IOMM-Lee cells infected with Ad-MMP-9 with or without irradiation (Fig. 5A). Further, in the presence of Dn-ERK the levels of phospho-ERK and Akt was inhibited in IOMM-Lee cells infected with Ad-MMP-9. Both the experiments clearly indicated that the phosphorylation of Akt is negatively regulated by inhibiting the phosphorylation of ERK (Fig. 5).
However, the total forms remain unaffected in controls and treated groups.

PI3K inhibitor decreases the phosphorylation of Akt and $E R K$. The impact of blocking phosphorylation of Akt on ERK was investigated by treating the Ad-MMP-9-infected cells with a PI3K inhibitor. Western blot analysis of the cell lysates indicated that the inhibitor interfered with the 
A
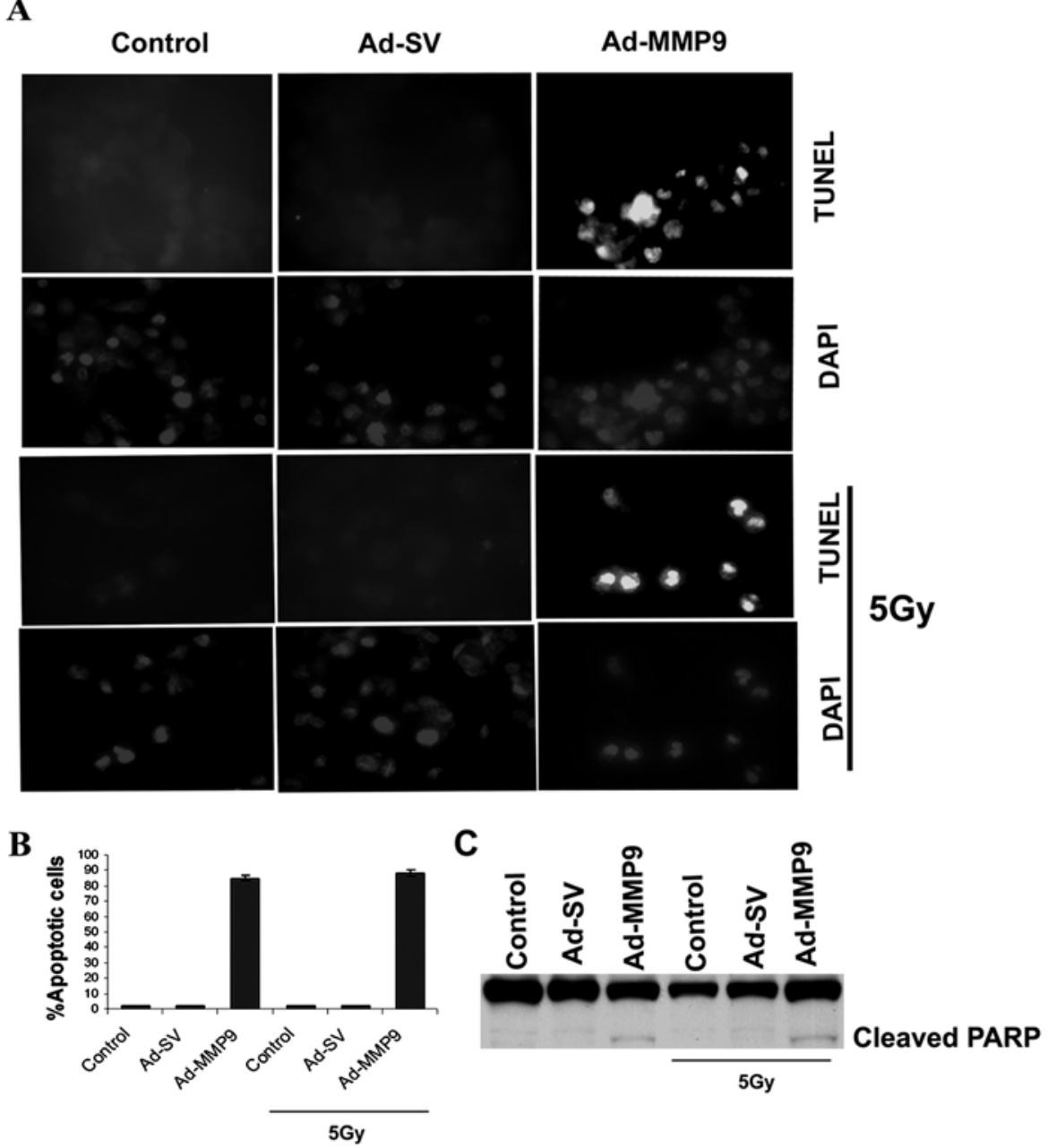

Figure 3. Ad-MMP-9 infection induces apoptosis in IOMM-Lee cells. (A) IOMM-Lee cells were infected with Ad-SV or Ad-MMP-9, irradiated with 5 Gy, and stained for apoptosis by TdT-mediated dUTP nick end-labeling assay (TUNEL). Data shown are from representative fields. (B) Cell death was quantified as percent of apoptotic cells against controls. Quantification of apoptotic cells indicated an increase in the number of TUNEL-positive cells per microscopic field in Ad-MMP-9-treated cells. Values are mean \pm SD from three different experiments (p<0.05). (C) Cell lysates from Ad-SV or Ad-MMP-9-infected and control groups were used for immunoblot analysis for PARP levels using specific antibodies.

phosphorylation of Akt. In addition, a decrease in the phosphorylation of ERK was also observed, thereby revealing the Akt-mediated modulation of ERK phosphorylation (Fig. 6A). As in the MEK1/2 treatment, the total forms of both ERK and Akt remained unaffected.

Ectopic expression of constitutively active Akt induces ERK phosphorylation. To further characterize the reciprocal influence of Akt phosphorylation on the activation of ERK, we transiently transfected the IOMM-Lee cells with myr-Akt for $48 \mathrm{~h}$. The cell lysates were subjected to Western blot analysis using antibodies against pERK and pAkt. The transfected cells exhibited a marked increase in the phosphorylation of Akt as well as ERK (Fig. 6B) compared to controls and Ad-SV infected cells.

Ad-MMP-9 executes apoptosis via a c-JUN and Fas-dependent mechanism. Since the activation of Akt in apoptosis has been reported thus far as a rare event, we studied the downstream molecules of Akt in the execution of apoptosis. As reported in the apoptosis of epidermal cells (21), the involvement of c-JUN and Fas were assessed. In the cells treated with Ad-MMP-9, total and phospho forms of c-JUN (Ser-73) were found to be decreased while Fas increased (Fig. 7). However, treatment with a PI3K inhibitor restored both the forms of c-JUN but the Fas levels were significantly decreased. Phospho-c-JUN (Ser-243), which is subjected to proteasomal degradation, was present more in Ad-MMP-9-treated cells; its levels were reduced in PI3K inhibitor-treated cells. The attenuation of apoptotic activity in the PI3K inhibitor-treated cells was confirmed by the absence of cleaved PARP (Fig. 7), which were infected with Ad-MMP-9 previously.

\section{Discussion}

Maximum surgical resection and adjuvant radiotherapy have been shown to be independent prognostic predictors for patients with meningiomas. However, optimum treatment modalities for meningiomas have not been well defined because of their surgically inaccessible location $(3,25)$, recurrence (4) as well as the problem of radiation-induced development of malignant meningiomas (10). Sublethal doses 
A

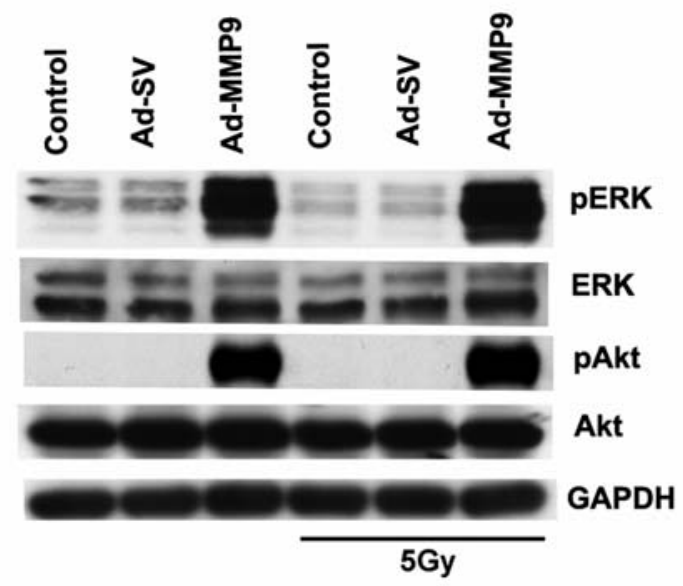

B

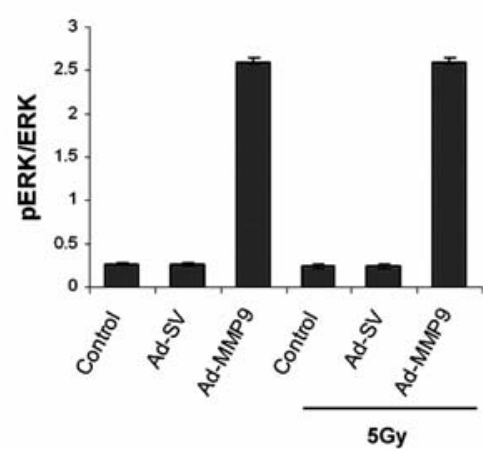

C

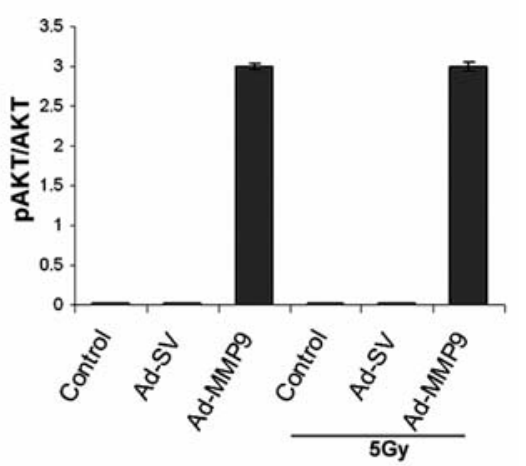

Figure 4. Ad-MMP-9 treatment leads to the activation of ERK and Akt. (A) IOMM-Lee cells were treated with 50 MOI of Ad-SV or Ad-MMP-9 for 24 h, after which one set of infected plates were irradiated at $5 \mathrm{~Gy}$. After $16 \mathrm{~h}$ of radiation, cell lysates were assessed for total and phospho-ERK and Akt levels by Western blot analysis using the respective antibodies. (B) Densitometric analyses of Western blots described in (A) for pERK/ERK (B) and pAkt/Akt (C). Results are expressed as a fold increase in phosphorylation compared with the mock control. Phosphorylation is calculated as ratios of the phosphorylated versus nonphosphorylated forms (means $\pm \mathrm{SD} ; \mathrm{n}=3$ ). Data are representative of three independent experiments $(\mathrm{p}<0.05)$. GAPDH was used as a loading control.

A

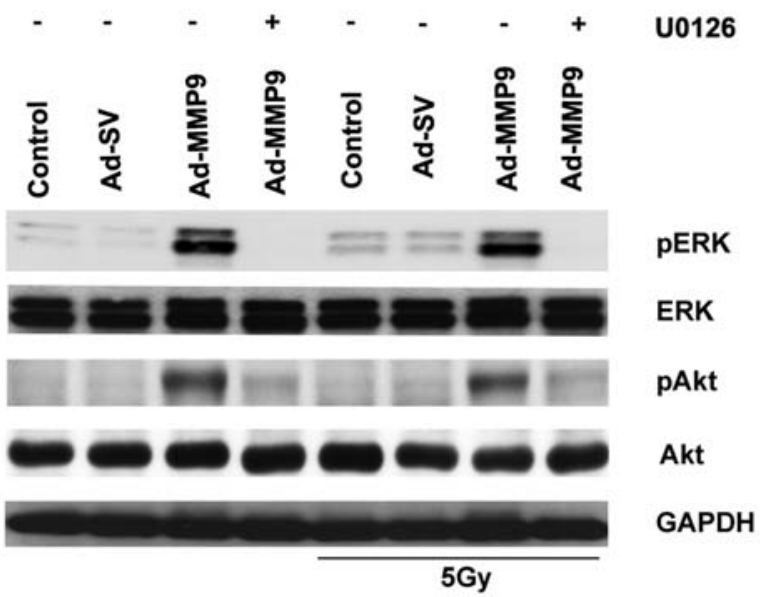

of radiation have the potential to augment cell proliferation and resistance because they are capable of activating growth factor receptors and subsequent pro-survival signaling pathways $(26,27)$. Ionizing radiation has been shown to induce the expression of MMP-1 and MMP-10 in squamous cell carcinoma (28), MMP-1 and MMP-3 in human dermal fibroblasts (29), and MMP-2 secretion in glioma cells (26). In this study, we report that the exposure of malignant meningioma IOMM-Lee cells to sublethal radiation leads to the overexpression of MMP-9. In concordance with our findings, radiation induced MMP-9 overexpression at mRNA and protein level were reported in hepatocellular carcinoma cell lines (12) and in irradiated BRE-SNB19 cocultures (30).
B

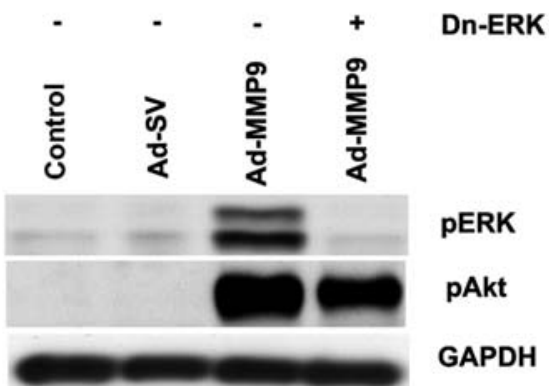

Figure 5. MEK1/2 inhibitor and Dn-ERK constructs attenuate the phosphorylation of ERK and Akt. (A) IOMM-Lee cells were infected with 50 MOI of Ad-SV or Ad-MMP-9 and then treated with U0126 $(20 \mu \mathrm{M})$. After $24 \mathrm{~h}$ of infection and treatment, one group of cells was irradiated at $5 \mathrm{~Gy}$. After $16 \mathrm{~h}$ of irradiation, cell lysates were analyzed for total ERK, Akt and phospho-ERK and Akt levels by Western blot analysis using the respective antibodies. GAPDH served as a loading control. (B) IOMM-Lee cells were transfected with $2 \mu \mathrm{g} / \mathrm{ml}$ of Dn-ERK plasmid and infected with 50 MOI Ad-SV or Ad-MMP-9. Cell lysates were subjected to Western blotting with phospho-specific antibodies of ERK and Akt. 
A

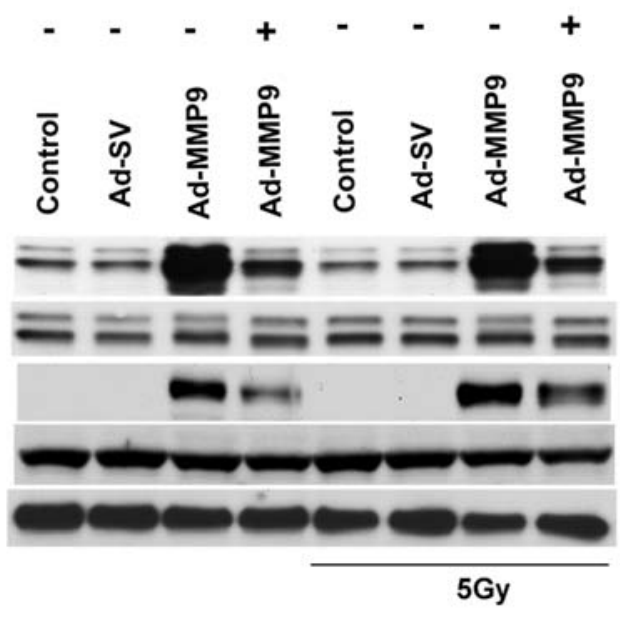

B

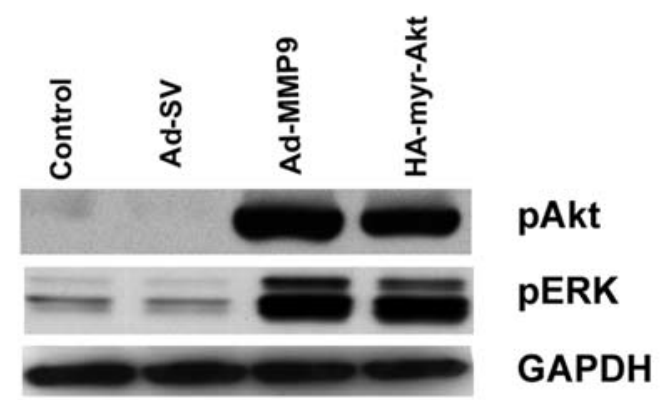

Figure 6. PI3K inhibitor blocks the activation of Akt and ERK and ectopic expression of active Akt activates ERK. (A) IOMM-Lee cells were infected with 50 MOI of Ad-SV or Ad-MMP-9 and then treated with LY294002 $(10 \mu \mathrm{M})$. After $24 \mathrm{~h}$ of infection and treatment, one group of cells was irradiated at $5 \mathrm{~Gy}$. After $16 \mathrm{~h}$ of irradiation, cell lysates were analyzed for total and active forms ERK and Akt by Western blot analysis using the respective antibodies. GAPDH served as a loading control. (B) IOMM-Lee cells were transfected with $2 \mu \mathrm{g} / \mathrm{ml}$ of HA myr-Akt plasmid and infected with 50 MOI of Ad-SV or Ad-MMP-9. Cell lysates were subjected to Western blotting with phospho-specific antibodies for ERK and Akt.

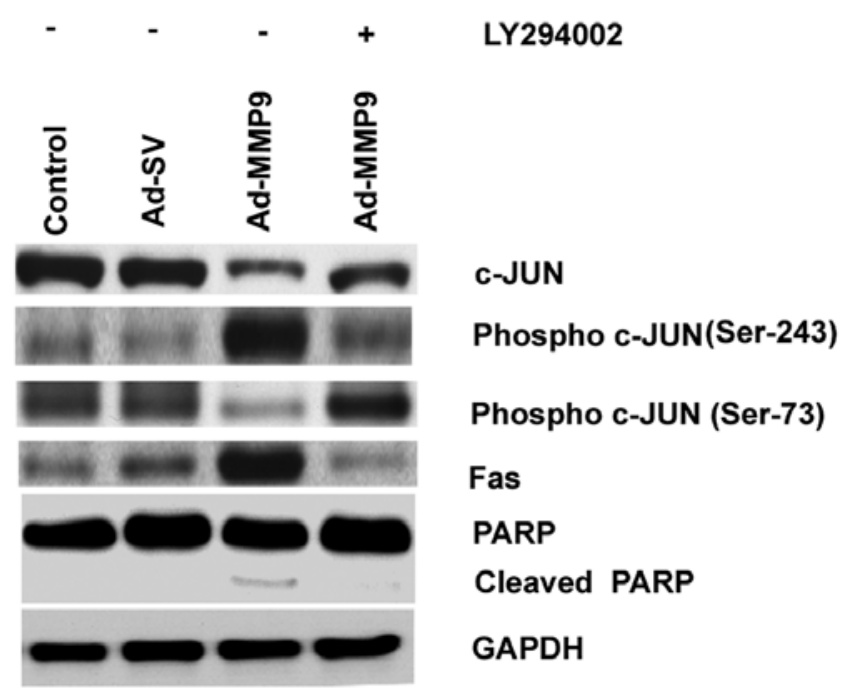

Figure 7. PI3K inhibitor attenuates apoptosis. Following infection with Ad-SV or Ad-MMP-9, IOMM-Lee cells were treated with $20 \mu \mathrm{M}$ of PI3K inhibitor. Equal quantities of protein as cell lysates were subjected to immunoblotting with antibodies specific for c-JUN, pc-JUN (ser 73), pc-JUN (ser 273), Fas and PARP. GAPDH was used as a loading control. Each blot is a representative of three independent experiments.
We also show the enhanced migration and invasiveness of IOMM-Lee cells during radiation treatment, which is in agreement with the studies of Wild-Bode et al $(31,32)$ and Hegedus et al (33) on glioma cell lines and Cheng et al on hepatocellular carcinoma (12). Furthermore, we showed that the aggressive tumor characteristics can be reversed using RNAi-mediated downregulation of MMP-9. Earlier studies by our group and others have demonstrated the correlation between MMPs and the invasiveness of different cancer cell lines (34-36). Consistent with our present results, several studies have shown that synthetic MMP inhibitors block the invasion of cancer cells by inhibiting MMP activation process $(37,38)$. In addition, inhibition of radiation-enhanced MMP-9 protein expression with antisense oligonucleotides of MMP-9 has been shown to significantly suppress radiationinduced invasion (39).

Our investigation revealed the apoptosis-inducing ability of Ad-MMP-9 in IOMM-Lee cells. We also show that the phosphorylation of ERK and Akt are key events in the apoptotic cells. Although the pro-survival and anti-apoptotic roles of ERK and Akt-mediated signaling cascades have been widely reported, evidence exists that the ERK-mediated apoptosis can be induced by diverse stimuli in different cell lines and tissues (40). Also, the Akt-mediated positive regulation of Fas-mediated apoptosis in epidermal cell death has been reported (41). Cross-talk among different intracellular signaling pathways have been generally adopted as strategies by the cancer cells under stress conditions. In this study, the use of ERK and PI3K specific inhibitors, transfection with a dominant negative ERK construct and ectopic expression of constitutively active Akt revealed an interdependent mechanism between ERK and Akt with one modulating the activity of the other. Consistent with these results, Ouabain-stimulated ERK phosphorylation has been shown to be required for Akt phosphorylation on Ser (473) in opossum kidney cells (42).

In agreement with the earlier studies on Akt-mediated apoptosis executed by c-JUN and Fas, our data also suggest that the inhibition of the PI3K pathway leads to the restoration of phospho-c-JUN (Ser-73), the decrement in the Fas, and eventually the rescue of cells from siRNA-caused apoptosis. Attenuation of apoptosis has not been observed in MEK1/2 inhibitor-treated experiments (data not shown)-probably because of the blockade of basal phosphorylation of ERK that is essential for cell survival.

In conclusion, our data show that radiation enhances MMP-9 expression and its associated aggressive characteristics in malignant meningioma cells. However, we also demonstrate that the siRNA-mediated knockdown of MMP-9 is effective in attenuating these properties. Furthermore, Ad-MMP-9 treatment induces apoptosis via the concomitant activation of ERK and Akt.

\section{Acknowledgements}

We thank Shellee Abraham for assistance in the manuscript preparation and Diana Meister and Sushma Jasti for the review. We also thank Professor Richard A. Roth for the construct encoding the constitutively active myristoylated AKT (myr-AKT $\delta$ 4-129). This research was supported by 
National Cancer Institute Grants CA 75557, CA 92393, CA 95058, CA 116708, N.I.N.D.S. NS47699, NS57529, NS061835, and Caterpillar, Inc., OSF Saint Francis, Inc., Peoria, IL (to J.S.R.).

\section{References}

1. Louis DN, Scheithauer BW, Budka H, von Deimling A and Kepes JJ: Meningiomas pathology and genetics of tumours of the nervous system. In: World Health Organization Classification of Tumours. Kleihues P and Cavenee WK (eds.), IARC Press, Lyon, pp176-184, 2000.

2. Barbaro NM, Gutin PH, Wilson CB, Sheline GE, Boldrey EB and Wara WM: Radiation therapy in the treatment of partially resected meningiomas. Neurosurgery 20: 525-528, 1987.

3. Goldsmith BJ, Wara WM, Wilson CB and Larson DA: Postoperative irradiation for subtotally resected meningiomas. A retrospective analysis of 140 patients treated from 1967 to 1990. J Neurosurg 80: 195-201, 1994

4. Kokubo M, Shibamoto Y, Takahashi JA, Sasai K, Oya N, Hashimoto N and Hiraoka M: Efficacy of conventional radiotherapy for recurrent meningioma. J Neurooncol 48: 51-55, 2000.

5. Goyal LK, Suh JH, Mohan DS, Prayson RA, Lee J and Barnett GH: Local control and overall survival in atypical meningioma: a retrospective study. Int J Radiat Oncol Biol Phys 46: 57-61, 2000.

6. Hug EB, Devries A, Thornton AF, Munzenride JE, Pardo FS, Hedley-Whyte ET, Bussiere MR and Ojemann R: Management of atypical and malignant meningiomas: role of high-dose, 3D-conformal radiation therapy. J Neurooncol 48: 151-160, 2000 .

7. Jaaskelainen J, Haltia M and Servo A: Atypical and anaplastic meningiomas: radiology, surgery, radiotherapy and outcome. Surg Neurol 25: 233-242, 1986.

8. Hovinga KE, Stalpers LJ, van BC, Donker M, Verhoeff JJ, Rodermond HM, Bosch DA and van Furth WR: Radiationenhanced vascular endothelial growth factor (VEGF) secretion in glioblastoma multiforme cell lines - a clue to radioresistance? J Neurooncol 74: 99-103, 2005

9. Sheng-Hua C, Yan-Bin M, Zhi-An Z, Hong Z, Dong-Fu F, Zhi-Qiang L and Xian-Hou Y: Radiation-enhanced hepatocyte growth factor secretion in malignant glioma cell lines. Surg Neurol 68: 610-613, 2007.

10. Choudhary A, Pradhan S, Huda MF, Mohanty S and Kumar M: Radiation induced meningioma with a short latent period following high dose cranial irradiation - case report and literature review. J Neurooncol 77: 73-77, 2006.

11. Osipov V, Ho KC, Krouwer HG, Meyer G and Shidham VB: Post-radiation dedifferentiation of meningioma into osteosarcoma. BMC Cancer 2: 34, 2002.

12. Cheng JC, Chou CH, Kuo ML and Hsieh CY: Radiationenhanced hepatocellular carcinoma cell invasion with MMP-9 expression through PI3K/Akt/NF-kappaB signal transduction pathway. Oncogene 25: 7009-7018, 2006.

13. Susskind H, Hymowitz MH, Lau YH, Atkins HL, Hurewitz AN, Valentine ES, Meek AG and Zucker S: Increased plasma levels of matrix metalloproteinase-9 and tissue inhibitor of metalloproteinase-1 in lung and breast cancer are altered during chest radiotherapy. Int J Radiat Oncol Biol Phys 56: 1161-1169, 2003.

14. Qian LW, Mizumoto K, Urashima T, Nagai E, Maehara N, Sato N, Nakajima M and Tanaka M: Radiation-induced increase in invasive potential of human pancreatic cancer cells and its blockade by a matrix metalloproteinase inhibitor, CGS27023. Clin Cancer Res 8: 1223-1227, 2002

15. Bhoopathi P, Chetty C, Kunigal S, Vanamala SK, Rao JS and Lakka SS: Blockade of tumor growth due to matrix metalloproteinase- 9 inhibition is mediated by sequential activation of beta1integrin, ERK and NF-kappaB. J Biol Chem 283: 1545-1552, 2008

16. Rao JS, Bhoopathi P, Chetty C, Gujrati M and Lakka SS: Matrix metalloproteinase-9 short interfering RNA induced senescence resulting in inhibition of medulloblastoma growth via p16INK4 and mitogen-activated protein kinase pathway. Cancer Res 67: 4956-4964, 2007

17. Choi BK, Choi CH, Oh HL and Kim YK: Role of ERK activation in cisplatin-induced apoptosis in A172 human glioma cells. Neurotoxicology 25: 915-924, 2004.
18. Kim YK, Kim HJ, Kwon CH, Kim JH, Woo JS, Jung JS and Kim JM: Role of ERK activation in cisplatin-induced apoptosis in OK renal epithelial cells. J Appl Toxicol 25: 374-382, 2005.

19. Gulbins E, Hermisson M, Brenner B, Grassme HU, Linderkamp O, Dichgans J, Weller M and Lang F: Cellular stimulation via CD95 involves activation of phospho-inositide-3-kinase. Pflugers Arch 435: 546-554, 1998.

20. Lu B, Wang L, Stehlik C, Medan D, Huang C, Hu S, Chen F, Shi X and Rojanasakul Y: Phosphatidylinositol 3-kinase/Akt positively regulates Fas (CD95)-mediated apoptosis in epidermal Cl41 cells. J Immunol 176: 6785-6793, 2006.

21. Tkacz K, Cioroch M, Skladanowski AC and Makarewicz W: The cytotoxic effect of purine riboside on COS-7 cells. Adv Exp Med Biol 486: 355-359, 2000.

22. Lakka SS, Jasti SL, Gondi CS, Boyd D, Chandrasekar N, Dinh DH, Olivero WC, Gujrati M and Rao JS: Downregulation of MMP-9 in ERK-mutated stable transfectants inhibits glioma invasion in vitro. Oncogene 21: 5601-5608, 2002.

23. Chomczynski P and Sacchi N: Single-step method of RNA isolation by acid guanidinium thiocyanate-phenol-chloroform extraction. Anal Biochem 162: 156-159, 1987.

24. Gondi CS, Lakka SS, Yanamandra N, Siddique K, Dinh DH, Olivero WC, Gujrati M and Rao JS: Expression of antisense uPAR and antisense uPA from a bicistronic adenoviral construct inhibits glioma cell invasion, tumor growth and angiogenesis. Oncogene 22: 5967-5975, 2003.

25. Cohen M and Trott NG: Radiology, physical science and the emergence of medical physics. Med Phys 22: 1889-1897, 1995.

26. Park CM, Park MJ, Kwak HJ, Lee HC, Kim MS, Lee SH, Park IC, Rhee $\mathrm{CH}$ and Hong SI: Ionizing radiation enhances matrix metalloproteinase-2 secretion and invasion of glioma cells through Src/epidermal growth factor receptor-mediated p38/Akt and phosphatidylinositol 3-kinase/Akt signaling pathways. Cancer Res 66: 8511-8519, 2006.

27. Valerie K, Yacoub A, Hagan MP, Curiel DT, Fisher PB, Grant S and Dent P: Radiation-induced cell signaling: inside-out and outside-in. Mol Cancer Ther 6: 789-801, 2007.

28. Ramos MC, Steinbrenner H, Stuhlmann D, Sies H and Brenneisen P: Induction of MMP-10 and MMP-1 in a squamous cell carcinoma cell line by ultraviolet radiation. Biol Chem 385: 75-86, 2004.

29. Brenneisen P, Wlaschek M, Schwamborn E, Schneider LA, Ma W, Sies H and Scharffetter-Kochanek K: Activation of protein kinase CK2 is an early step in the ultraviolet B-mediated increase in interstitial collagenase (matrix metalloproteinase-1; MMP-1) and stromelysin-1 (MMP-3) protein levels in human dermal fibroblasts. Biochem J 365: 31-40, 2002.

30. Nirmala C, Jasti SL, Sawaya R, Kyritsis AP, Konduri SD Ali-Osman F, Rao JS and Mohanam S: Effects of radiation on the levels of MMP-2, MMP-9 and TIMP-1 during morphogenic glial-endothelial cell interactions. Int J Cancer 88: 766-771, 2000.

31. Wild-Bode C, Weller M and Wick W: Molecular determinants of glioma cell migration and invasion. J Neurosurg 94: 978-984, 2001.

32. Wild-Bode C, Weller M, Rimner A, Dichgans J and Wick W: Sublethal irradiation promotes migration and invasiveness of glioma cells: implications for radiotherapy of human glioblastoma. Cancer Res 61: 2744-2750, 2001.

33. Hegedus B, Zach J, Czirok A, Lovey J and Vicsek T: Irradiation and Taxol treatment result in non-monotonous, dose-dependent changes in the motility of glioblastoma cells. J Neurooncol 67: 147-157, 2004.

34. Kunigal S, Lakka SS, Gondi CS, Estes N and Rao JS: RNAimediated downregulation of urokinase plasminogen activator receptor and matrix metalloprotease- 9 in human breast cancer cells results in decreased tumor invasion, angiogenesis and growth. Int J Cancer 121: 2307-2316, 2007.

35. Pazzaglia L, Ponticelli F, Magagnoli G, Magagnoli G, Gamberi G, Ragazzini P, Balladelli A, Picci P and Benassi M: Activation of metalloproteinases-2 and -9 by interleukin-1alpha in S100A4positive liposarcoma cell line: correlation with cell invasiveness. Anticancer Res 24: 967-972, 2004.

36. Tsung AJ, Kargiotis O, Chetty C, Lakka SS, Gujrati M, Spomar DG, Dinh DH and Rao JS: Downregulation of matrix metalloproteinase-2 (MMP-2) utilizing adenovirus-mediated transfer of small interfering RNA (siRNA) in a novel spinal metastatic melanoma model. Int J Oncol 32: 557-564, 2008. 
37. Jimenez RE, Hartwig W, Antoniu BA, Compton CC, Warshaw AL and Fernandez-Del CC: Effect of matrix metalloproteinase inhibition on pancreatic cancer invasion and metastasis: an additive strategy for cancer control. Ann Surg 231: 644-654, 2000.

38. Zervos EE, Shafii AE, Haq M and Rosemurgy AS: Matrix metalloproteinase inhibition suppresses MMP-2 activity and activation of PANC-1 cells in vitro. J Surg Res 84: 162-167, 1999.

39. London CA, Sekhon HS, Arora V, Stein DA, Iversen PL and Devi GR: A novel antisense inhibitor of MMP-9 attenuates angiogenesis, human prostate cancer cell invasion and tumorigenicity. Cancer Gene Ther 10: 823-832, 2003.
40. Zhuang S and Schnellmann RG: A death-promoting role for extracellular signal-regulated kinase. J Pharmacol Exp Ther 319: 991-997, 2006.

41. Ivanov VN, Krasilnikov M and Ronai Z: Regulation of Fas expression by STAT3 and c-Jun is mediated by phosphatidylinositol 3-kinase-AKT signaling. J Biol Chem 277: 4932-4944, 2002.

42. Khundmiri SJ, Amin V, Henson J, Lewis J, Ameen M, Rane MJ and Delamere NA: Ouabain stimulates protein kinase B (Akt) phosphorylation in opossum kidney proximal tubule cells through an ERK-dependent pathway. Am J Physiol Cell Physiol 293: C1171-C1180, 2007. 